\title{
Forecasting Stock Price using LSTM-CNN Method
}

\author{
Dinesh Reddy, Abhinav Karthik
}

\begin{abstract}
Foreseeing assumes an indispensable part in setting an exchanging methodology or deciding the ideal opportunity to purchase or sell stock. We propose an element combination long transient memory-convolutional neural organization (LSTM-CNN) model, which joins highlights gained from various presentations of similar information, i.e., stock timetable and stock outline pictures, to anticipate stock costs. The proposed model is created by LSTM and CNN, which extricate impermanent and picture components. We assessed the proposed single model (CNN and LSTM) utilizing SPDR S\&P 500 ETF information. Our LSTM-CNN combination highlight model surpasses single models in foreseeing evaluating. Also, we track down that the candle graph is the most precise image of a stock diagram that you can use to anticipate costs. Subsequently, this examination shows that prescient mistake can be viably decreased by utilizing a blend of transitory and picture components from similar information as opposed to utilizing these provisions independently.
\end{abstract}

Keywords: LSTM, CNN, RNN, STOCK MARKET.

\section{INTRODUCTION}

The change in stock prices has long been identified as a significant problem in the economic sector [5]. Stock costs are influenced by inward and outer inconstancy factors, like the homegrown and unfamiliar financial climate, worldwide circumstance, mechanical acknowledgment, finance information for recorded organizations, just as securities exchange execution. The conventional analysis method is based on economics and finance, which uses most of the primary analysis methods and methods of technical analysis. On the other hand, the direct way of research is very focused on internal stocks. It analytically analyzes external factors affecting the stock, such as interest rates, exchange rate, inflation, industrial policy, listed currencies companies, international relations, and other economies, as well as political factors. On the other hand, technical analysis focuses mainly on stock price controls, trading volume, and academic expectations of investors, which focuses on index trajectory analysis of individual or total stock markets using the Kline chart and other tools. Traditional fundamental and

Manuscript received on August 30, 2021.

Revised Manuscript received on September 09, 2021.

Manuscript published on October 30, 2021.

* Correspondence Author

Dinesh Reddy*, Department of Computer Science Engineering, SRM Institute of Science and Technology, Kattankulathur, India.

Abhinav Karthik, Department of Computer Science Engineering, Manipal Institute of Technology, Manipal, India

(C) The Authors. Published by Blue Eyes Intelligence Engineering and Sciences Publication (BEIESP). This is an open access article under the CC BY-NC-ND license (http://creativecommons.org/licenses/by-nc-nd/4.0/)

technical analyses are methods commonly used in many organizations and individual investors [6,11]. The accuracy of traditional fundamental analysis the way is hard to convince. Reason - is the only reason that influential factors are in the long-term cycle, but predictive results are highly dependent on the professional quality of the analysts. Like a series of financial periods, stock data have characteristics of random movement [10]. Some scholars use the time direct prediction model for short-term guessing based on statistics and probability of existence. Stock price with many long-term details autoregressive integrated intermediate mobile mode, and single heteroskedasticity model with independent conditions. However, the accuracy of using the model alone time series is in question due to the uncertainty and height of the sound features of the financial timeline series. The relationship between independent and dependent variables tends to fluctuate over time, i.e., reduces its continuous performance and expansion [11]. Financial timeline series data can be used as numerical and image data to predict stock prices. DNNs, especially convolutional neural networks (CNNs), can read or extract these features themselves. CNN stands out with in-depth reading strategies in the field of computer viewing, object discovery, segmentation, and so on. The examinations expected to work on the exactness of foreseeing objective qualities by blending information from various sorts of information or different assets as opposed to perusing one-dimensional information. The correct construction built from different presentations will learn the dynamic features and the other characteristics of each building, improving the accuracy of the prediction. From this impetus, in this examination, we propose a combination model that joins CNN and LSTM to consolidate ents of different introductions from monetary time series information to Further develop precision in foreseeing value levels. We say this proposed model is a LSTM-CNN highlight model. This model reads chart patterns and shows the temporal features contained in the timeline series data. fore building an LSTM-CNN integration model, we mus each model tailored to this specific data representation. (emaining learning constructions and ontainer conses from stock graph pictures to make CNN advanced for stock outline pictures. At last, we assembled the LSTM-CNN include model The proposed model we call the LSTM-CNN highlight model. When training the LSTM-CNN feature model, we use integrated training to demonstrate each training process simultaneously to increase the efficiency of the proposed model. 
In this investigation, we utilize the moment minutes of the SPDR S\&P 500 ETF Trust (SPY) tick information as the information of the monetary course of events series since it has the most elevated exchanging volume between ETF markets. Using this data, we created various presentations to fit our models. We created four stock chart images to see which idea is best for predicting prices.

The primary donation of this paper are as follows:

1. Timeliness of a progression of stock value information, a new inside and out examination strategy (CNN-LSTM) is proposed to foresee stock costs. Thusly, CNN is utilized to remove the information component, and LSTM is utilized to demonstrate information. It can exploit the course of events of stock value information to get more solid expectations.

2. By contrasting CNN-LSTM test pointers and multi-facet perceptron CNN, LSTM, and CNN-LSTM, it is demonstrated that CNN-LSTM has high prescient exactness and is exceptionally appropriate for stock value expectations.

\section{RELATED THEORY AND TECHNOLOGY ON RNN\&GRU}

\section{A. Recurrent Neural Networks}

Recurrent Neural Networks (RNN) are networks of neuron-like nodes arranged in sequential layers, with structures similar to normal Neural Networks. Neurons are divided into three layers, they are input layer, hidden layer, and output layer in Neural Networks. The connection between each neuron has interrelated trainable weight. Each neuron has a duty to a fixed time set. Neurons present in Hidden layer are leading in a time-dependent way. Everyone is totally connected only with the neurons that are present in hidden layer the give time step and are connected in a one-way link to every neuron allocated to later time step. Input and output neurons are only connected to the hidden layers by a step of the same allotted time. The input of the following time step is part of output of hidden layer of one-time step, the initiation of neurons is done in this time order: at every certain time step, only the neurons given to that time step calculates their activation.

\section{B. Architecture}

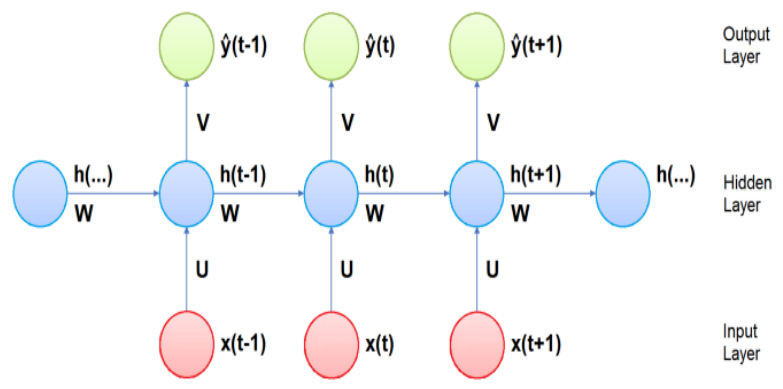

Figure 1:Architecure of RNN

Weights - In the Recurrent neural networks(RNN), the hidden layer neurons at the time $t$ is linked to the input vector of the given time $t$ by a weight matrix $U$, hidden layer neurons are linked to the neurons of time $(\mathrm{t}+1)$ and $(\mathrm{t}-1)$ by the weight matrix $\mathrm{W}$, and the output vector of time $(\mathrm{t})$ are interconnected to the hidden layer neurons by the weight matrix V; all the weight matrices are continuous for all time steps.

Input - The assumed vector $\mathrm{x}(\mathrm{t})$ is the network input at time step (t).

Hidden state - For time(t), Vector $\mathrm{h}(\mathrm{t})$ is the hidden state which is a place of the memory of the network; it is considered based on:

$$
h(t)=\tanh \left(W h(t-1)+U_{x}(t)\right)
$$

Output - The vector $y^{\wedge}(t)$ is an output of the network at a given time $t$ :

$$
\hat{y}(t)=\operatorname{softmax}(V s(t))
$$

\section{Learning algorithm}

The essential objective of the arrangement cycle is to track down the best weight networks of $\mathrm{U}, \mathrm{V}$, and $\mathrm{W}$ that gives the best gauge of $\mathrm{y}^{\wedge}(\mathrm{t})$, starting from the information $\mathrm{x}(\mathrm{t})$, of the genuine worth $\mathrm{y}(\mathrm{t})$. To achieve this, we indicated a target work called the loss function and it is signified by the letter $\mathrm{J}$, which assesses the scale between the genuine and the figure esteems on a preparation set. The beneath condition gives it.

$$
J(\theta)=\frac{1}{m} \sum_{i=1}^{m} \sum_{t=1}^{N_{i}} L(\hat{y}(t), y(t))
$$

Were,

- the cost function L evaluates the scale between the original and forecasted estimates on a specific time step.

- Here $\mathrm{m}$ is considered as the dimension of the training set. Here $\theta$ is considered as the vector of standard parameter. The loss function $\mathrm{J}$ is decreased by utilizing these two huge strides: forward propagation and in backward propagation through time. These means are duplication ordinarily, and the quantity of duplications is called epoch number. Forward propagation - With the proper boundaries $\mathrm{U}, \mathrm{W}$, and $\mathrm{V}$, information is proliferated through the organization, and at every second $t$, we process $y^{\wedge}(t)$ utilizing the recently characterized recipes. Eventually, the misfortune work is determined.

\section{DIFFERENT TYPES OF RNN}

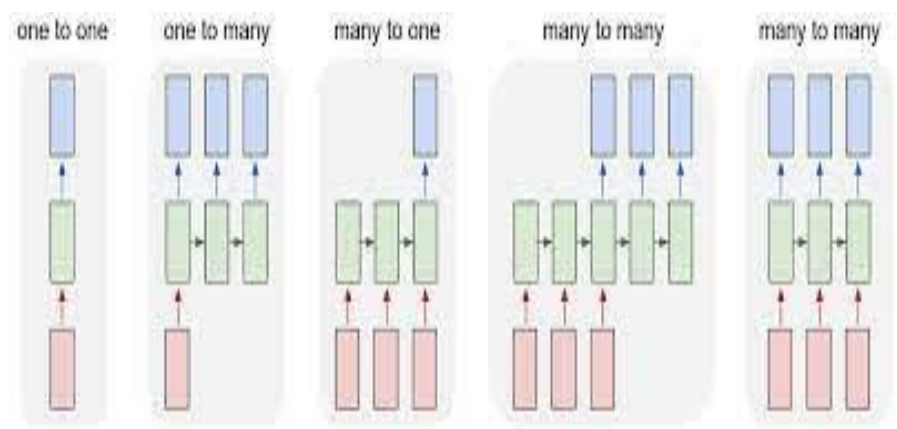

Figure 2: Different types of RNN

- Image classification

- Sequence output

- Sequence input

- Sequence output Sequence input

- Synced Sequence output and input

Published By:

Blue Eyes Intelligence Engineering and Sciences Publication (BEIESP)

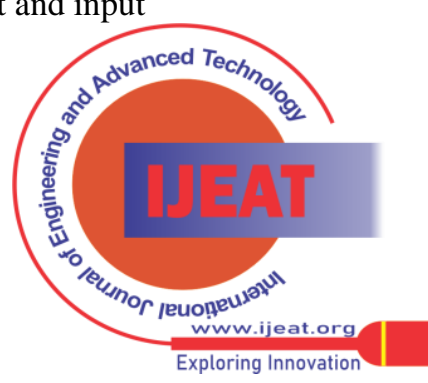




\section{GATED RECURRENT UNIT (GRU)}

The GRU is another time of Recurrent Neural Organizations (RNN) and like LSTM. To tackle the vanish angle issue of a standard RNN, GRU utilizes the update gate and reset gate. These are two gates that choose what information ought to be passed into the output. These two gates can be told to keep data from many strides before the genuine time step, without washing it through the time, or eliminate superfluous data for the forecast. On the off chance that painstakingly prepared, GRU can perform astoundingly well, even in complex situations. As displayed beneath Figure, a GRU unit is made out of:

- a reset gate that chooses how much data from the past time step can be neglected.

- an update gate that chooses how much data from the past time steps should be saved.

- a memory that carries data with the whole arrangement and shows the memory of the organization.

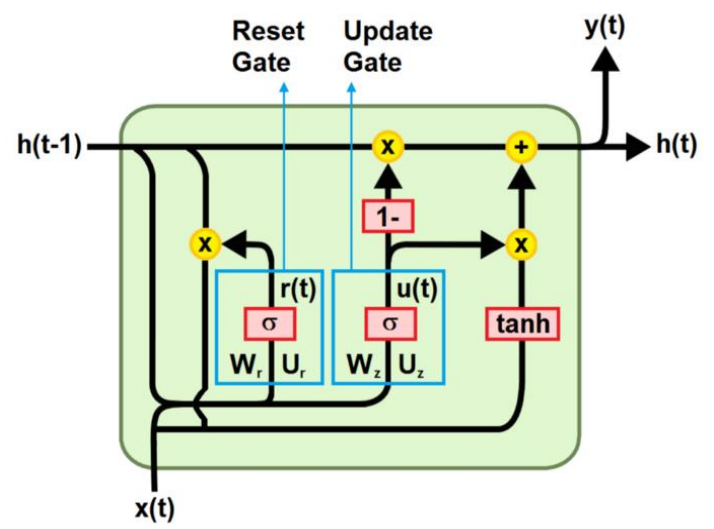

Figure 3: Architecture of GRU

\section{A. Reset Gate}

The fundamental gate is the reset gate. It decides how to get the new information together with the past memory, choosing how much data from past time steps can be neglected. First and foremost, the weighted sum between the input $\mathrm{x}(\mathrm{t})$ and the memory $\mathrm{h}(\mathrm{t}-1)$, which holds the data before $\mathrm{t}-1$ stages, is performed. Then, at that point the sigmoid activation work is applied to squash the outcome somewhere in the range of 0 and 1 .

$$
r(t)=\sigma\left(x(t) U_{r}+h(t-1) W_{r}\right)
$$

\section{UPDATE GATE}

The subsequent gate is the update gate. It assists the model with deciding the amount of the data from past time steps should be given to what's to come. That is incredible in light of the fact that the model can choose to duplicate all the data from an earlier time and dispense with the danger of the evaporating inclination issue. The recipe to work out it is like one for the reset gate. In any case, the distinction comes in the loads and the gate's use (it will be evident in computing the memory).

$$
z(t)=\sigma\left(x(t) U_{z}+h(t-1) W_{z}\right)
$$

\section{PROPOSED PREDICTION MODEL}

Building up a stock file value estimating model has three stages: data collection and preprocessing, mode establishment and training, and evaluation of experiment results, as displayed. The LSTM- Attention network structure hidden layer includes a LSTM, consideration, and weighty

\section{Data source}

We chose three significant stock files as exploratory information: the Dow Jones Modern Normal (DJIA), 500 index (S\&P 500), and Hang Seng index (HSI). The S\&P 500 and Dow Jones Modern Normal (DJIA) information are from the date January 2000 to July 2019. The Hang Seng Index (HSI) information are from January 2002, to July, 2019.S\&P This information is from Kaggle! Finance. There are six factors in the essential monetary dataset. The initial value (open) is the main monetary cost per portion of safety afte the market opens on the exchanging day, and the end value (close) is its last cost on that day. High is the greatest cost of a stock exchanges a day, and low is the least cost. Change close is the end cost after changes for detach and profit dispersions. Information has been changed utilizing fitting detach and profit multipliers. Volume alludes to the quantity of exchanges in a specific time unit for promoting.

\section{Data preprocessing}

We carry out the suggested stock estimating technique in Python utilizing TensorFlow. We utilize zero-mean standardization to the information and partitions it into preparing and test datasets. For the (S\&P 500) and (DJIA) informational collections, information from January 2000 to May 2019, is utilized for model preparing, and information from May 17, 2019, to July 1, 2019, were utilized for testing. For the Hang Seng Index (HSI) informational index,information from January 2002 to May 2019, is utilized for preparing, and from May 2019 to July 2019, for testing. Because of the confounded and unstable financial exchange and different exchanging constraints, the stock costs we see are brash.[12] All the while, the monetary time series is fixed and displays the covering of sound signals and commotion, which makes conventional denoising fruitless. The wavelet change is more appropriate for exceptionally unpredictable monetary groupings since it can break down both the time and recurrence domains.[13] It amalgamates with the regular hypothesis of time series examination that shows great relevance. Henceforth, wavelet evaluation has turned into a useful asset to deal with monetary time series information. [14] We utilize a wavelet change with multiscale attributes to denoise the dataset, viably isolating the significant sign from the commotion. Especially, we utilize the coif3 wavelet work with three deterioration layers, and we assess the aftereffect of the wavelet change by the signal-to-noise ratio (SNR). Root mean square errors (RMSE). The more noteworthy the SNR and the more modest the RMSE, the better the denoising impact of the wavelet change: 


$$
\mathrm{SNR}=10 \log \left[\frac{\sum_{\mathrm{j}-1}^{\mathrm{N}} \mathrm{x}^{2}}{\sum_{-1}^{\mathrm{N}}\left(\mathrm{x}_{\mathrm{j}}-\bar{x}_{j}\right)^{2}}\right] .
$$

\section{EXPERIMENTS}

To demonstrate the viability of CNN-LSTM, we contrasted this strategy and CNN, LSTM, and LSTM-CNN utilizing similar preparing set and testing set information under a similar working space.

\section{A. Data.}

In this analysis, the Dow Jones Industrial Average (DJIA) is chosen as the exploratory information. The day by day exchanging information of 2768 exchanging days from January 2009 to December 2019, are gotten from the Kaggle dataset. Each piece of information contains seven things, to be specific, Date, Open, High, Low, Close, Adj Close, Volume. The following day's end cost is anticipated.

\section{B. Model Implementation.}

We select three diverse execution gauges, the root mean absolute error (RMAE), the root mean square error (RMSE), and the MAPE, to assess the estimate force of our proposed models. While we train models, we will utilize RMSE as a misfortune work, which implies that the model is prepared to lessen RMSE. RMSE is an ideal measure for uncovering moderately enormous estimate blunders. RMAE is helpful for showing the orderly inclination of the model, and MAPE is a proportion of the precision of forecasts in measurements. These conditions are as per the following:

$$
\begin{aligned}
\text { RMAE } & =\sqrt{\frac{1}{N} \sum_{i=1}^{N}\left|x_{1, i}-x_{2, i}\right|} \\
\text { RMSE } & =\sqrt{\frac{1}{N} \sum_{i=1}^{N}\left(x_{1, i}-x_{2, i}\right)^{2}} \\
\text { MAPE } & =\frac{100}{N} \sum_{i=1}^{N}\left|\frac{x_{2, i}-x_{1, i}}{x_{1, i}}\right|
\end{aligned}
$$

\section{LONG-SHORT TERM MEMORY (LSTM)}

LONG-SHORT TERM MEMORY is an artificial intelligent recurrent network (RNN) architecture. RNN allows information to be continuously looped into the network and, thus, persist previous information. At every loop in the RNN, some info is 'drop out' as output, and some information loops back into the network. The following figure illustrates a simple RNN architecture.

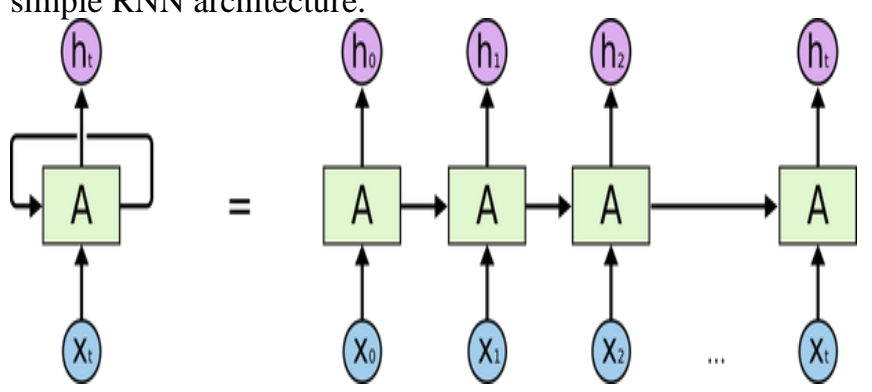

Figure 4: Simple RNN architecture

LSTM is a type of RNN that is efficient of learning long-term dependency. Due to the property, it is robust in processing, classifying, and predicting time series data. The following tangent tanh as the activation function.

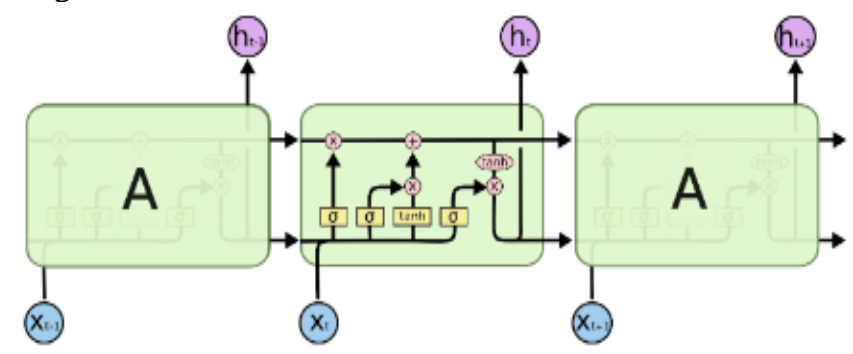

Figure 5: Simple LSTM model

\section{Data preparation}

To normalize the data, we take the algorithm of the ratio of two consecutive daily closing values

$$
\log \left(\frac{C \operatorname{los} e_{t}}{C \operatorname{los} e_{t-1}}\right)
$$

In terms of prediction, we use previous 30 days data to predict the Close index at day $\mathrm{t}+35$. Combine with data normalization; the following sequence is an input for the LSTM mode:

$$
\log \left(\frac{C \operatorname{los} e_{t}}{C \operatorname{Cos} e_{t-1}}\right), \log \left(\frac{C \operatorname{lose} e_{t+1}}{C \log e_{t}}\right), \log \left(\frac{C \operatorname{los} e_{t+2}}{C \log e_{t+1}}\right), \log \left(\frac{C \operatorname{los} e_{t+3}}{C \operatorname{los} e_{t+2}}\right), \log \left(\frac{C \operatorname{los} e_{t+4}}{C \operatorname{los} e_{t+3}}\right) \ldots
$$

The data set downloaded from Yahoo Finance does not value, so a process for missing value is not needed. The data set is divided into two groups: training and test set with ratio 8:2. To normalize the data, we take the algorithm of the ratio of two consecutive daily closing values.

\section{Model input}

The model's input is the sequence of logs shown above.

\section{Model output}

The model's output is the prediction for log.

\section{Implementation of the LSTM model}

The LSTM model is implanted on Google Colab using Python 3 and Keras library for deep learning. The implementation code includes three main parts.

The first part implements data processing and normalization, including loading Dow Jones Data set with panda's library. Data normalization is done with logarithm functions in the NumPy library. For the second part, the model's architecture and hyper-parameters are defined. The LSTM model contains two smaller parts: a single layer and three entirely linked layers. The output from the single layer is then passed through the entirely linked layers. The important hyper-parameters of the LSTM model include dropout rate, number of epochs, regularize and batch size. After trial-and-error optimization, the following hyper-parameters produce the lowest error for the Dow Jones data set: dropout rate for all layers of 0.8 , numbers, number of epoch equal 20,12 regularize, 'Adam' optimizer, and batch size of

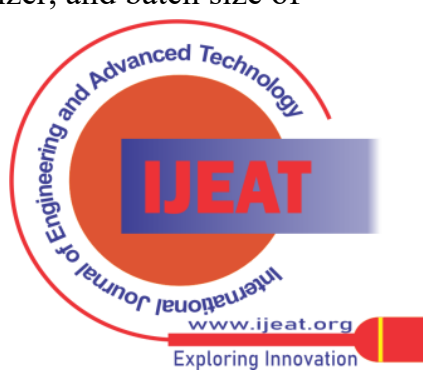




\section{LSTM MODEL RESULT(Training)}

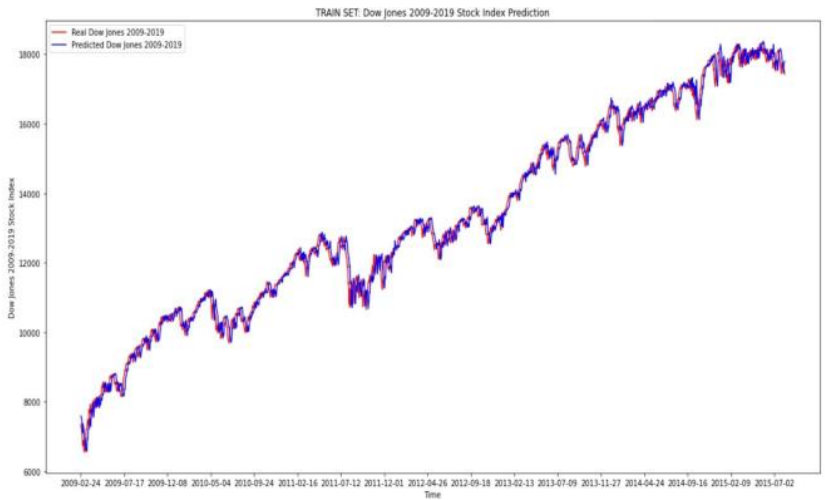

Figure 6: Plot of LSTM model for the training set RMSE $=$ 263.228, $M A E=208.75$

\section{LSTM MODEL RESUL (TESTING)}

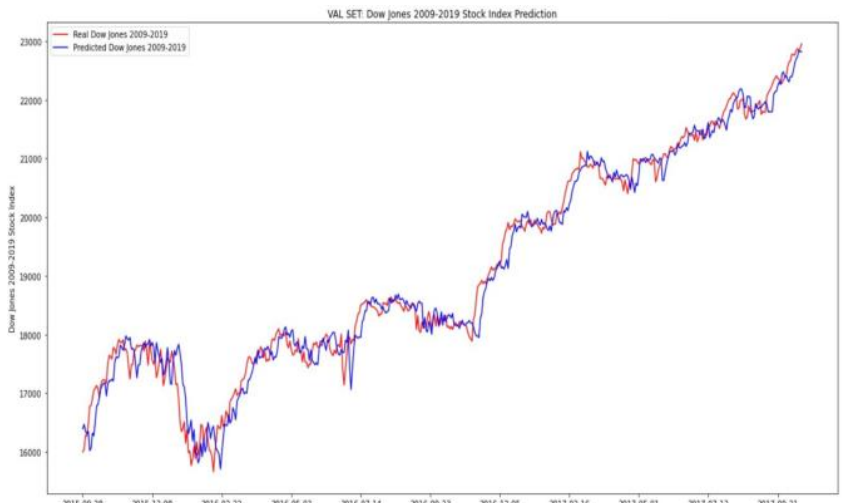
RMSE=296.74, MAE $=228.32$

\section{Model Evaluation}

The root mean square error (RMSE) of the training, validation, and test data is $251,271,520$, respectively. Due to the stochastic nature of the data, if predicting for a shorter period, the prediction's error will decrease. In terms of run time, the model entirely runs under 1 minute, which is reasonable for deployment. The following plot shows the LSTM fit for the training and testing data set.

\section{CONVOLUTIONAL NEURAL NETWORK (CNN)}

Convolutional Neural Network is quite similar to neural networks like Perceptron or Multi-Layer Perceptron docs. The difference here is we don't need to flatten the input image (that creates a large number of weights). For instance, if we have the input image with size 112 x 112, we need to take 12544 operations for weights. With CNN, the neurons only have a small set of weights ( $5 \times 5=25$, with 5 is kernel size) that were applied a whole bunch of small subsets of the image of the same size. It takes in the 'neighborhood' highlights found in the recently covered up layer instead of pixel pictures. In addition, the last yield layer will diminish the full picture into a single vector of output value. Convolutional Neural network start to lead the pack of the way that the information contains pictures, and they oblige the design all the more reasonably. In contrast to a normal Neural Organization, the layers of a CNN have neurons masterminded in 3 measurements: width, tallness, depth. In this undertaking, we use picture measurements $112 \times 112 \times 3$ (width, height, depth)
Figure 7: The plot of the LSTM model for the test set

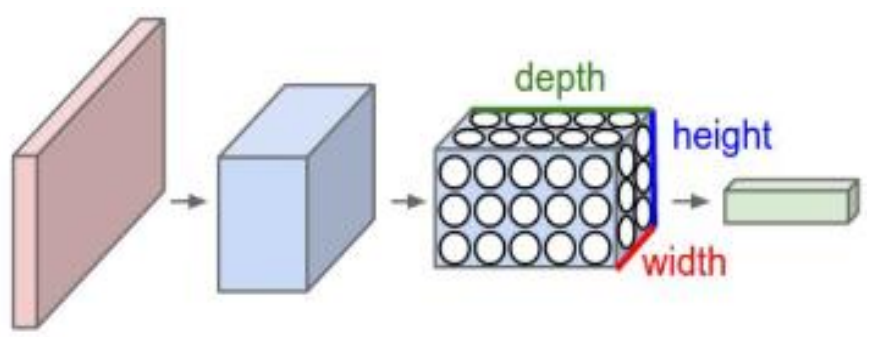

Figure 8: CNN

Left: 3 General Neural Network. Right: ConvNet coordinates its neurons into three sizes (width, height, depth), as displayed in one of the layers. All ConvNet layers convert the $3 \mathrm{D}$ information volume into the $3 \mathrm{D}$ yield volume of the neuron startup. In this given model, the red information layer holds the picture, so the width and tallness can be the size of the picture, and the profundity will be 3 (Channels red, green, blue).

\section{Residual block of ResNet}

In the CNN model, we use three residual blocks that optimize for data like the stock chart.
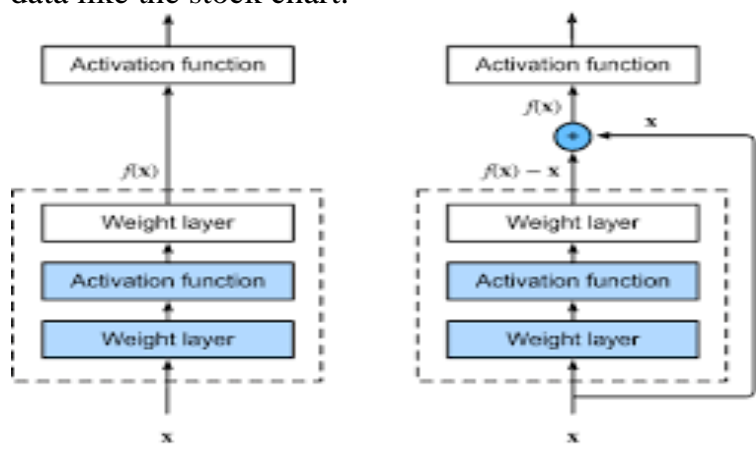

Figure 9: Residual block of ResNet

Figure 9: The right figure outlines the lingering square of ResNet, where the strong line conveying the layer input $x$ to the expansion administrator is known as residual connection (or easy route association). With leftover squares, data sources can advance spread quicker through the lingering associations across layers.

\section{Model input}

Image size $112 \times 112$ with RGB channels $(112 \times 112 \times 3)$ that shows the movement of price in the time interval between $\mathrm{t}-30$ and $\mathrm{t}$
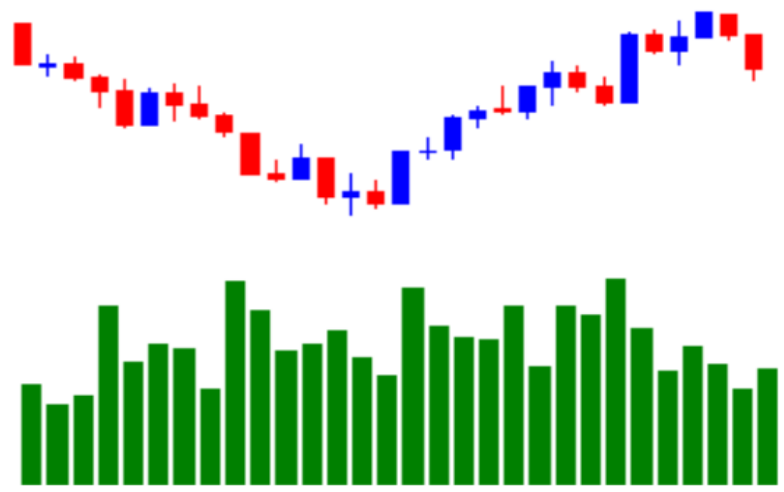

Figure 10: Time interval between $t-30$ and $t$

Published By:

Blue Eyes Intelligence Engineering and Sciences Publication (BEIESP)

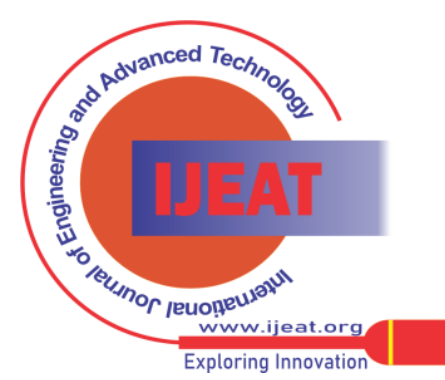




\section{Model output}

Predicted Close price of $\mathrm{t}+5$

\section{Purpose of project}

Economic time series information can be utilized as numeric information and as picture information that is changed in anticipating the stock costs. Specialized examination utilizes the outline pictures to foresee stock costs by discovering designs in them. In spite of the fact that using an engineering built from the various portrayals of similar information will become familiar with similar provisions and different attributes of every design, which can further develop the forecast exactness. From this inspiration, we need to check CNN out.

\section{Advantages and disadvantages of the CNN model:}

The main advantage of CNN that makes them suited to forecasting time series is the ability to use filtrate to compute dilations between each cell, allowing the neural network to understand better the relationship between the different observations in the time series. However, this is just an assumption, and it takes more time and computational resources to train the model.

\section{DATA PREPARATION}

Convert time series data to an image. Each image shows the movement of price in the time interval between $t-30$ and $t$. Target Value is the closing price of $\mathrm{t}+5$

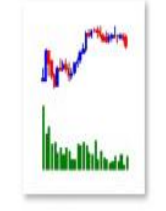

candlebar 0-29.p

$\mathrm{ng}$

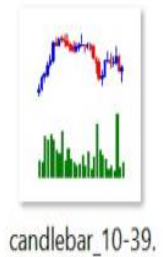

png

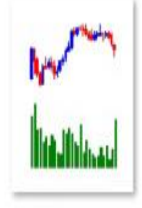

candlebar_1-30.p

$\mathrm{ng}$

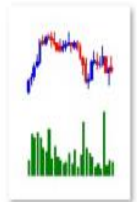

candlebar_11-40

png

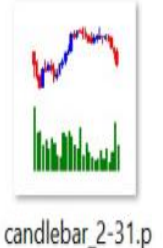

$\mathrm{ng}$

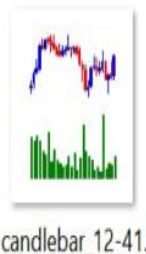

png

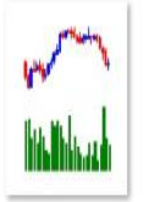

candlebar $3-32$. p

$\mathrm{ng}$

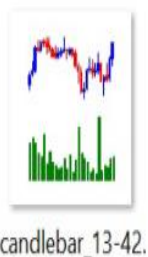

png

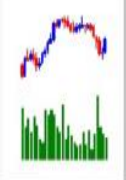

candlebar 4-33.p

$\mathrm{ng}$

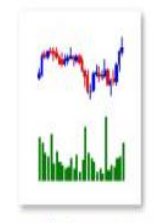

candlebar_14-43

png
Figure 11: time interval between $t-30$ and $t$. Target Value is the closing price of $t+5$

EVALUATE THE MODEL CNN MODEL RESULT(Training)

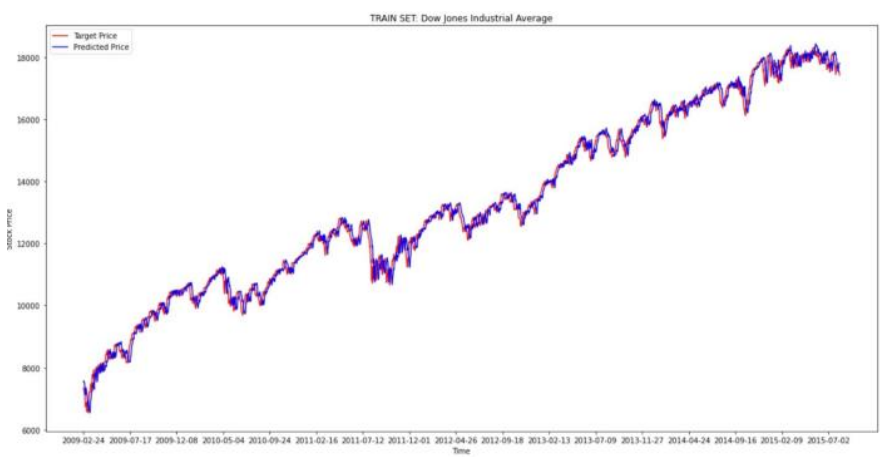

Figure 12: CNN model on Training set
$\mathrm{RMSE}=250.69, \mathrm{MAE}=191.699$

\section{CNN MODEL RESULT(Testing)}

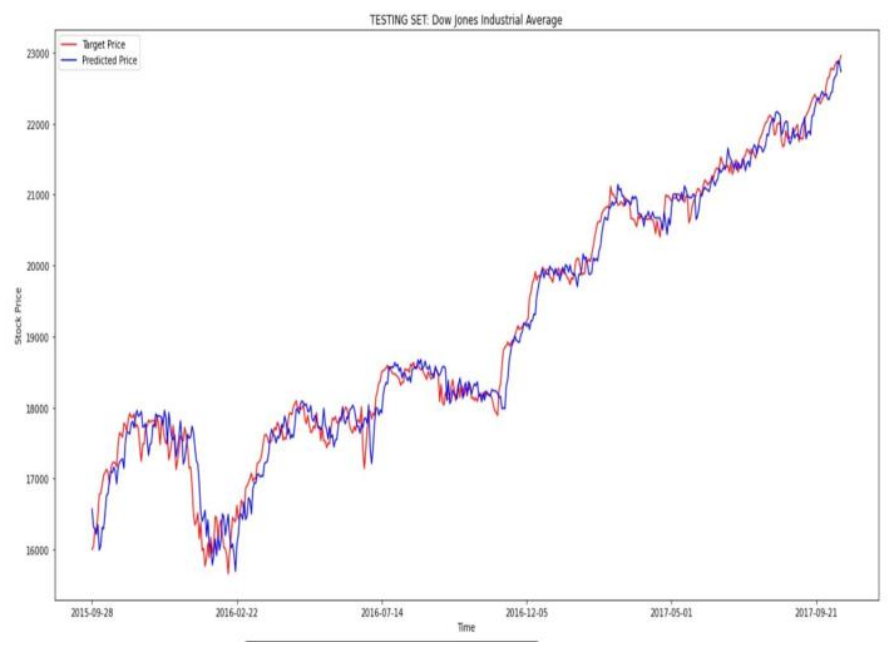

Figure 13: CNN model on Testing set

$\mathrm{RMSE}=270.61, \mathrm{MAE}=201.36$

\section{LSTM-CNN MODEL DEFINITION}

We take the combination of picture and worldly elements from linking each part and each different trait, like picture and material qualities. It is made out of three stages. To start with, CNN is prepared to lessen CNN misfortune. This interaction includes learning a graphical provisions of the diagram picture. Second, LSTM is acquainted with limit LSTM misfortune. In this cycle, the worldly quality is combination LSTM-CNN shares the boundaries contained in convolutional layers in CNN and LSTM layers in LSTM.

\section{MODEL INPUT}

Time series and candlebar chart for a time period between $\mathrm{t}-30$ and $\mathrm{t}$ of price

\section{MODEL OUTPUT}

Predicted Close Price of $\mathrm{t}+5$

\section{ADVANTAGES AND DISADVANTAGES OF THE LSTM-CNN MODEL}

We expect that the combination of the various components containing the extricated stock graph pictures and stock time-series information from similar information can further develop the preparation model. Practically speaking, it gives a little preferable outcome over each different model.

\section{DATA PREPARATION}

We have two data streams for each type of data-time series and an image chart. The preparation is similar to LSTM and CNN models.

\section{EVALUATE THE MODEL}

\section{LSTM-CNN MODEL RESULT(Training)}

\section{Published By:}

Blue Eyes Intelligence Engineering and Sciences Publication (BEIESP)

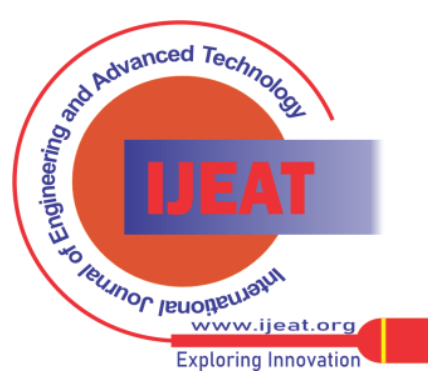




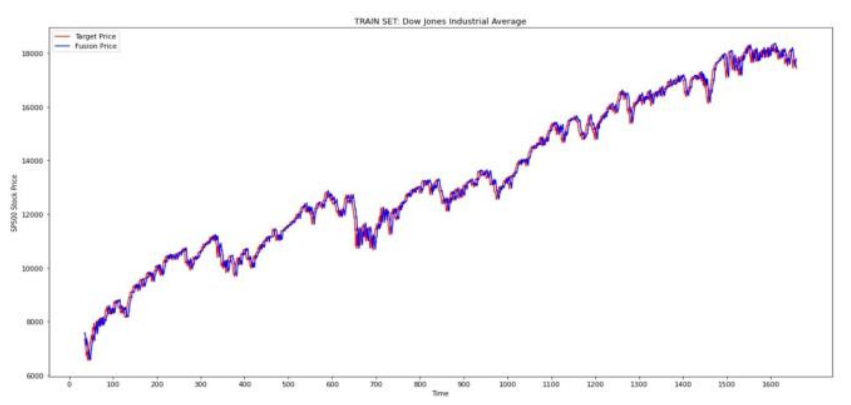

Figure 14: LSTM-CNN model on Training set RMSE $=251.21$ MAE $=191.477$

\section{LSTM-CNN MODEL RESULT(Testing)}

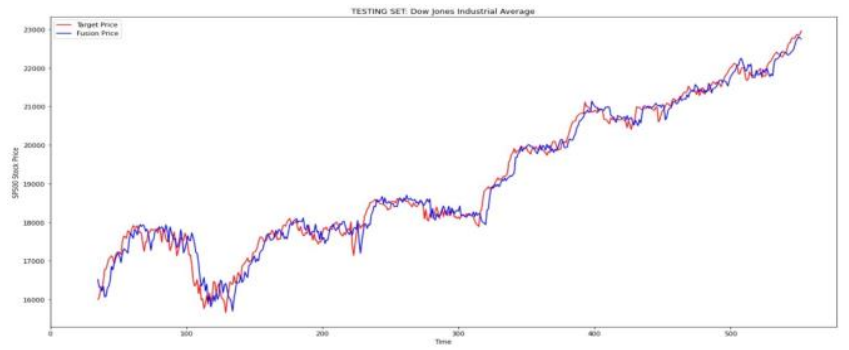

Figure 14: LSTM-CNN model on Testing set RMSE $=$ 267.56 MAE $=198.29$

\section{MODEL COMPARISON}

Result on Dow Jones Industrial Average (DJIA) (test set)

\section{RMSE MAE}

\begin{tabular}{lll} 
LSTM & 296.7456 & 228.3258 \\
CNN & 270.6161 & 201.3691 \\
LSTM-CNN & 269.0664 & 198.8619 \\
\hline
\end{tabular}

Figure 15: Model Comparison

\section{CONCLUSION}

As far as the stock value arrangement information, the paper proposes CNN-LSTM to anticipate the following day's stock shutting cost. This strategy utilizes the initial value, the most exorbitant cost, the least value, the end value, volume, benefit, variances, and the transformation of stock information as info, completely using the course of events elements of the stock information. CNN is utilized to separate the information highlights. LSTM is utilized to peruse the yield information and foresee the stock shutting sum the following day. - The paper takes the significant subtleties of the Shanghai Composite Record as an illustration to approve the test outcomes. - e Test outcomes show that CNN-LSTM has higher prescient exactness and preferable execution over CNN and LSTM and CNN-RNN. MAE and RMSE are the minor strategies close to 1 . CNN-LSTM is prepared at stock costs and can give important direction to financial backers to boost speculation benefits. CNN-LSTM likewise offers the experience of directing a populace put together overview with respect to the subtleties of the monetary course of events series. Notwithstanding, the model actually has a few blemishes. For instance, it just glances at the impact of stock stock estimate acknowledgement.

\section{REFERENCES} 1:309. help? J Forecast. 1998;17(1):59-80. Posted: 2007 prediction.

value information on shutting costs and neglects to incorporate enthusiastic factors like news and public arrangement into the estimate. Our future exploration work is essentially to grow the feeling examination of stock-related issues and government rules to guarantee the exactness of the

\section{APPENDIX}

It is optional. Appendixes, if needed, appear before the

\section{ACKNOWLEDGMENT}

It is optional. The preferred spelling of the word "acknowledgement" in American English is without an "e" after the "g." Use the singular heading even if you have many acknowledgements. Avoid expressions such as "One of us (S.B.A.) would like to thank ... ." Instead, write "F. A. Author thanks" Sponsor and financial support acknowledgements are placed in the unnumbered footnote on the first page.

1. Blume L, Easley D, Hara M. Market Statistics and Technical Analysis: The Role of Volume. J Finance [Internet]. 1994;49(1):153-81.

2. Taylor MP, Allen. The use of technical analysis in foreign exchange market. J Int Money Finacc. 1992;11(3):304-14.

3. Brown DP, Jennings RH. On Technical Analysis. Rev Finacc Stud

Cowles A. Can Stock Market Forecasters Forecast? Economy. 1933; Work. J Finance [Internet]. 1970;25(2):383-417.

Brooks C. Predicting the stock index volatility: Can market volume

International Journal of Computer Applications, 124 (12) (2015), pp.

. Ashish Sharma, Dinesh Bhuriya, Upendra Singh. "Survey of Stock Market Prediction Using Machine Learning Approach", ICECA 2017.

10. 1. VivekKanade, Bhausaheb Devikar, SayaliPhadatare, PranaliMunde, ShubhangiSonone. "Stock Market Prediction: Using Historical Data

1. Neural network modeling for stock movement prediction, state of art

12. Chavan, P. S., \& Patil, S. T. (2013). Parameters for stock market

. International Journal of Computer Technology and Applications, 4(2),

Permet Index using Neura

\section{AUTHORS PROFILE}

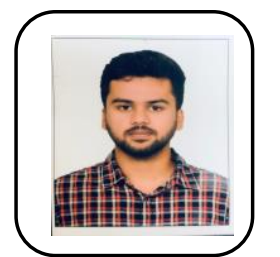
related to problem-solving and Data science in his motatidineshreddy663@gmail.com

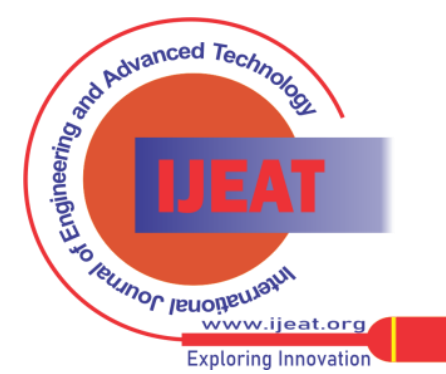


Forecasting Stock Price using LSTM-CNN Method

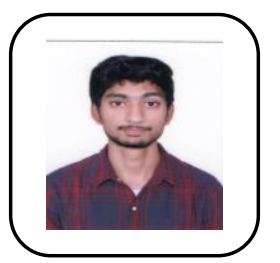

Pulikonda Abhinav Karthik, is currently pursuing his B.Tech. from Dept. of Computer Science \& Engineering at Manipal Institute of technology, Manipal, India. He has attended workshops and induction programs conducted by various organizations has done Banking system project in Java. His areas of research interest are data analytics and machine learning. This is his first

paper on machine learning and its algorithms. Email: abhinavkarthik29@gmail.com

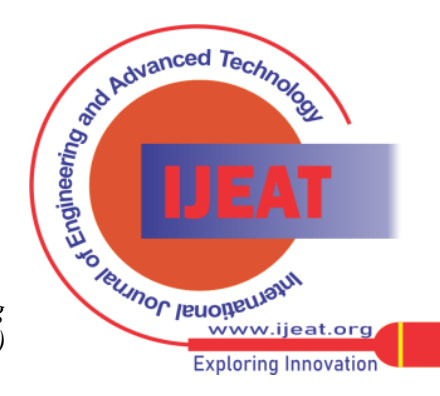

